\title{
Adubação orgânica, nutrição e progresso de cercosporiose e ferrugem-do-cafeeiro
}

\author{
Florisvalda da Silva Santos(1), Paulo Estevão de Souza ${ }^{(2)}$, Edson Ampélio Pozza(2), Júlio César Miranda(2), \\ Eudes Arruda Carvalho(2), Luiz Henrique Monteiro Fernandes ${ }^{(2)}$ e Adélia Aziz Alexandre Pozza ${ }^{(3)}$
}

(1)Universidade Estadual da Bahia, Campus IX, CEP 48000-000 Barreiras, BA. E-mail: florsantos@uneb.br (2)Universidade Federal de Lavras (Ufla), Departamento de Fitopatologia, Caixa Postal 3037, CEP 37200-000 Lavras, MG, E-mail: pauleste@ufla.br, eapozza@ufla.br, jcmiranda@ufla.br, eudesa@ufla.br, luizh_agronomia@hotmail.com (3)Ufla, Departamento de Ciência do Solo. E-mail: alana@ufla.br

Resumo - O objetivo deste trabalho foi avaliar o efeito de fontes nutricionais orgânicas, no progresso da cercosporiose e da ferrugem-do-cafeeiro, entre novembro/2003 e novembro/2005. Utilizou-se o delineamento de blocos ao acaso, com quatro repetições e seis tratamentos: palha de café + chorume suíno (PC+CS); torta de mamona + crotalária $(\mathrm{TM}+\mathrm{Cr})$; palha de café $(\mathrm{PC})$; esterco bovino + crotalária $(\mathrm{EB}+\mathrm{Cr})$; palha de café + torta de mamona (PC+TM); e composto orgânico, torta de mamona, chorume suíno e crotalária (testemunha). A maior área abaixo da curva de progresso da incidência da cercosporiose e da ferrugem foi registrada no tratamento PC (respectivamente 62 e $38 \%$ superiores à testemunha). $\mathrm{O}$ maior progresso das doenças coincidiu com a elevação no teor de $\mathrm{K}$ e redução nos teores de $\mathrm{Ca}$ foliares no tratamento $\mathrm{PC}$, comparado às demais fontes de adubação, e resultou em maior desfolha e menor produtividade. Os tratamentos $\mathrm{PC}+\mathrm{TM}$ e $\mathrm{PC}+\mathrm{CS}$ reduziram a incidência da cercosporiose em $38 \%$ e da ferrugem em 31 e $21 \%$, respectivamente, e aumentou o teor de Ca foliar ao final da fase de granação do cafeeiro, em comparação ao tratamento PC. O equilíbrio nutricional desses cafeeiros conferiulhes maior resistência e reduziu o efeito da bienalidade nas safras 2003/2004 e 2004/2005.

Termos para indexação: Cercospora coffeicola, Hemileia vastatrix, epidemiologia, fontes.

\section{Organic fertilization, nutrition and the progress of brown eye spot and rust in coffee trees}

\begin{abstract}
The objective of this work was to evaluate the effect of organic nutrional sources on the progress of brown eye spot and coffee rust in coffee plants, between November 2003 and November 2005. The experimental design adopted was the randomized blocks, with four replications and six treatment plots: coffee fruit peel + swine waste $(\mathrm{CP}+\mathrm{SW})$; castor bean presscake + crotalaria $(\mathrm{CBP}+\mathrm{Cr})$; coffee fruit peel $(\mathrm{CP})$; bovine manure + crotalaria $(\mathrm{BM}+\mathrm{Cr})$; coffee fruit peel + castor bean presscake $(\mathrm{CP}+\mathrm{CBP})$; and standard fertilization used in the farm, which combines compost, castor bean presscake, swine waste and crotalaria (control). The higher area under the incidence progress curves of brown eye spot and coffee rust was obtained by CP treatment (respectively 62 and 38\% higher to control). The larger progress coincided with the increase in leaf potassium and decrease of calcium content in $\mathrm{CP}$, compared to other nutrient sources, and it resulted in higher leaf drop and lower yield. $\mathrm{CP}+\mathrm{CBP}$ and $\mathrm{CP}+\mathrm{SW}$ reduced in $38 \%$ the brown eye spot incidence, and in 31 and $21 \%$, respectively, the coffee rust incidence, and increased leaf calcium content during fruit filling, compared to CP. The better nutritional status of plants increased resistance to diseases and reduced the variation between 2004 and 2005 harvests.
\end{abstract}

Index terms: Cercospora coffeicola, Hemileia vastatrix, epidemiology, source.

\section{Introdução}

A cercosporiose (Cercospora coffeicola Berk. \& Cooke) e a ferrugem (Hemileia vastatrix Berkeley \& Broome) são as principais doenças em cafezais brasileiros e ocasionam perdas na produtividade, estimadas entre 35 e 40\% na ausência de medidas de controle (Acuña et al., 1998; Carvalho \& Chalfoun, 1998). O progresso das doenças é favorecido por vários fatores relacionados ao patógeno, ao hospedeiro e ao ambiente. Entre esses, o desequilíbrio nutricional entre o potássio e o cálcio torna severa a ocorrência da cercosporiose em cafeeiros, no sistema convencional (Carvalho \& Chalfoun, 1998; Pozza et al., 2001; Garcia Júnior et al., 2003), adubados com fontes minerais prontamente solúveis ou sob irrigação (Talamini et al., 2001). 
Em cafeeiros conduzidos no sistema orgânico, a mineralização e a lenta liberação de nutrientes podem promover o desequilíbrio nutricional temporário durante o ciclo produtivo, o que favorece o progresso da cercosporiose e da ferrugem. Embora esse sistema utilize práticas de manejo, responsáveis por favorecer a expressão dos mecanismos de defesa das plantas, e embora favoreçam as populações de inimigos naturais dos patógenos (Bruggen, 1995), algumas vezes essas práticas não são suficientes, para impedir que ocorram epidemias em cultivares suscetíveis. Há registros de incidência de $40 \%$ de cercosporiose e de $50 \%$ de ferrugem, em lavouras de café orgânico na Malásia (Phiri et al., 2001). Na Costa Rica, Samayoa-Juaréz \& Sánchez-Garcia (2000) verificaram incidência de cercosporiose e de ferrugem acima de 20 e $40 \%$, respectivamente. Em lavouras de café orgânico em Minas Gerais, Martins et al. (2004) registraram incidência de cercosporiose acima de 59\%. Relatos como esses são reconhecidos, por técnicos e cafeicultores, como o principal ponto de estrangulamento na produtividade do cafeeiro orgânico. Embora o preço obtido na comercialização desse tipo especial de café seja maior, com exportações que alcançaram preço superior a $\mathrm{R} \$ 500,00$ a saca, quando o preço do produto convencional esteve em R $\$ 170,00$ (Embrapa, 2004), geralmente, sua produtividade é inferior à do café convencional. Tais perdas podem ser agravadas pela intensa desfolha, causada por ambas as doenças, e pela queda prematura de frutos, principalmente quando a cercosporiose incide nas fases iniciais da maturação.

Além das perdas ocasionadas por doenças, na maioria das vezes a cafeicultura orgânica não consegue se autosustentar e, para obter níveis de produtividade economicamente viáveis, grandes quantidades de adubo orgânico precisam vir de fontes externas à lavoura. Diferentes tipos de resíduos de origem vegetal e animal, em diferentes estádios de decomposição, podem ser utilizados para adubar o cafeeiro orgânico. A escolha dos resíduos a serem aplicados no cafeeiro depende de sua disponibilidade. Porém, a eficiência no fornecimento de nutrientes está diretamente relacionada à sua taxa de decomposição, que é influenciada por diversos fatores, dos quais se destaca a relação entre as quantidades de carbono e nitrogênio (relação C:N) de sua composição (Ricci et al., 2005).

Em razão da grande quantidade e variedade de fontes de adubação e do conhecimento sobre a estreita relação entre nutrição de plantas e sua suscetibilidade às doenças, é possível que uma determinada fonte de adubação possa afetar o progresso das doenças do cafeeiro, em ausência de agroquímicos. O presente estudo teve como objetivo avaliar o efeito de fontes nutricionais comumente utilizadas na adubação orgânica, no progresso da cercosporiose e da ferrugem-docafeeiro.

\section{Material e Métodos}

O estudo foi conduzido em lavoura de café orgânico, sem irrigação, na fazenda Cachoeira, em Santo Antônio do Amparo, MG, durante os anos agrícolas 2003/2004 e 2004/2005. O sistema de produção da Fazenda Cachoeira possui certificado orgânico desde 18/09/97, de acordo com as normas técnicas do Instituto Biodinâmico (IBD). O experimento foi conduzido em área experimental a $1.021 \mathrm{~m}$ de altitude, a $20^{\circ} 53^{\prime} 26^{\prime \prime} \mathrm{S}$ e $44^{\circ} 57^{\prime} 26^{\prime \prime} \mathrm{W}$, em solo caracterizado como Latossolo Vermelho Eutróficos típicos (Embrapa, 2006). Foram utilizados cafeeiros da cultivar Acaiá MG/474-19, com oito anos, no início do experimento, dispostos em espaçamento de plantio $2 \times 1 \mathrm{~m}$.

O delineamento foi o de blocos ao acaso, com quatro repetições e parcelas experimentais com 77 plantas. Dez cafeeiros, cujo enfolhamento e cargas pendentes foram os mais uniformes possíveis, foram selecionados para constituir a unidade experimental. Em todos os tratamentos (Tabela 1) as adubações foram feitas com

Tabela 1. Descrição dos tratamentos e quantidades das fontes nutricionais orgânicas aplicadas, nos anos agrícolas 2003/2004 (ano I) e 2004/2005 (ano II).

\begin{tabular}{|c|c|c|}
\hline \multirow[t]{2}{*}{ Tratamento } & \multicolumn{2}{|c|}{ Quantidade } \\
\hline & Ano I & Ano II \\
\hline Palha de café + chorume suíno $(\mathrm{PC}+\mathrm{CS})$ & $\mathrm{PC}=4,0+\mathrm{CS}=36,0 \mathrm{~L} \mathrm{planta}^{-1}$ & $\mathrm{PC}=9,0+\mathrm{CS}=45,0 \mathrm{~L} \mathrm{planta}^{-1}$ \\
\hline Torta de mamona + Crotalaria juncea $(\mathrm{TM}+\mathrm{Cr})$ & $\mathrm{TM}=5,0+$ L planta $^{-1}$ & $\mathrm{TM}=4,5 \mathrm{~L}_{\text {planta }}^{-1}$ \\
\hline Palha de café (PC) & $\mathrm{PC}=5,0 \mathrm{~L}_{\text {planta }}{ }^{-1}$ & $\mathrm{PC}=10,0 \mathrm{~L}_{\text {planta }}{ }^{-1}$ \\
\hline Esterco bovino $+C$. juncea $(\mathrm{EB}+\mathrm{Cr})$ & $\mathrm{EB}=15,0 \mathrm{~L}_{\text {planta }}{ }^{-1}$ & $\mathrm{~EB}=16,0 \mathrm{~L}_{\text {planta }}{ }^{-1}$ \\
\hline Palha de café + torta de mamona (PC+TM) & $\mathrm{PC}=4,0+\mathrm{TM}=2,0 \mathrm{~L}_{\text {planta }}^{-1}$ & $\mathrm{PC}=10,0+\mathrm{TM}=2,0 \mathrm{~L}_{\text {planta }}{ }^{-1}$ \\
\hline $\begin{array}{l}\text { Testemunha }=\text { composto orgânico }(\mathrm{CO})+\mathrm{TM}+ \\
\mathrm{CS} \text { ou farinha de ossos e carne }+\mathrm{Cr}\end{array}$ & $\mathrm{CO}=4,7+\mathrm{TM}=1,1+\mathrm{CS}=0,8 \mathrm{~L}_{\text {planta }^{-1}}$ & $\begin{array}{l}\mathrm{CO}=5,5+\mathrm{TM}=0,5+\mathrm{FOC}=0,1 \mathrm{~L}_{\text {planta }}^{-1}+ \\
\text { sulfato de potássio }=12 \mathrm{~g}^{-1} \text { planta }^{-1}\end{array}$ \\
\hline
\end{tabular}


base na dose de nitrogênio recomendada, em razão da produtividade esperada, e com base na análise da matéria orgânica das fontes (Tabela 2) e nos resultados da análise do solo (Tabela 3). Os adubos foram aplicados em uma única vez, sobre a superfície do solo, no mês de novembro. O adubo verde Crotalaria juncea foi cultivado com 27 sementes $\mathrm{m}^{-1}$ linear, em duas linhas de plantio, nas entrelinhas dos cafeeiros, em novembro e, aos 70 dias foi cortado e incorporado ao solo. Nenhum outro biofertilizante foi aplicado às parcelas. Nos dois anos de estudo, foram feitas pulverizações foliares de Viça-café plus, em cinco aplicações de novembro a março, à dose de $3 \mathrm{~kg} \mathrm{ha}^{-1}$, em toda a área experimental, e foram feitas capinas manuais entre as linhas de cafeeiro.

Foram avaliadas, quinzenalmente, entre novembro/ 2003 e novembro/2005, as incidências de cercosporiose e de ferrugem, em 12 folhas por planta, do terceiro ao quarto par, em ramos plagiotrópicos do terço médio de cafeeiros, escolhidos aleatoriamente, em amostragem não destrutiva. A variável incidência foi estimada a partir do número de folhas com lesões, dividido pelo número

Tabela 2. Composição química das fontes de adubação. Média de duas análises químicas.

\begin{tabular}{lcccccr}
\hline Fontes & $\mathrm{N}$ & $\mathrm{P}$ & $\mathrm{K}$ & $\mathrm{Ca}$ & $\mathrm{Mg}$ & $\mathrm{C} / \mathrm{N}$ \\
& $----------------(\%)$ & ----------------- & \\
\hline Chorume suíno (CS) & 3,4 & 5,5 & 0,6 & 3,0 & 0,7 & 5 \\
Torta de mamona (TM) & 5,6 & 2,1 & 1,5 & 6,4 & 3,7 & 6 \\
Palha de café (PC) & 1,5 & 0,3 & 2,4 & 0,6 & 0,1 & 30 \\
Esterco bovino (EB) & 1,1 & 0,4 & 1,3 & 0,4 & 0,1 & 25 \\
\hline
\end{tabular}

total de folhas avaliadas, tendo-se obtido a incidência média em cada parcela. A cercosporiose também foi avaliada em 200 frutos, no estádio "cereja", coletados em cada lado da linha de plantio, no terço médio das plantas de cada unidade experimental. As amostras foram encaminhadas para avaliação da incidência, estimada pela percentagem de frutos lesionados, e avaliação da severidade, estimada por uma escala de notas que representaram a área lesionada do fruto: 0 , fruto sem sintoma; 1,1 a 25\% da área lesionada; 2,26 a 50\% da área lesionada; $3,>50 \%$ da área lesionada (Boldini, 2001).

Os teores nutricionais dos cafeeiros foram avaliados por amostragem feita no terceiro par de folhas, na altura média da copa, nos dois lados das plantas, o que totalizou 100 folhas por unidade experimental, em três épocas, durante o desenvolvimento dos frutos: estádio inicial de desenvolvimento (fase de "chumbinho"), estádio "verde" (fase de expansão ou granação dos frutos), e próximo ao ponto de colheita (fase de maturação). As amostras de folhas foram encaminhadas para análises dos teores de nitrogênio, fósforo, potássio, cálcio, magnésio e enxofre, na matéria seca (Malavolta et al., 1997).

O enfolhamento foi estimado de duas maneiras. Avaliou-se, quinzenalmente, a ausência de folhas no terceiro e quarto pares de folhas de ramos plagiotrópicos, do terço médio dos cafeeiros escolhidos para avaliar as doenças, pela estimativa do percentual médio de desfolha por unidade experimental. Avaliou-se, mensalmente, o índice de área foliar (IAF) dos cafeeiros, por meio do

Tabela 3. Fertilidade do solo nas parcelas experimentais, nos anos agrícolas 2003/2004 e 2004/2005. Valores médios de quatro repetições ${ }^{(1)}$.

\begin{tabular}{|c|c|c|c|c|c|c|c|c|c|c|c|c|c|c|}
\hline Tratamento & $\begin{array}{c}\mathrm{pH} \\
\left(\mathrm{H}_{2} \mathrm{O}\right) \\
\end{array}$ & \multicolumn{2}{|c|}{$\begin{array}{c}\mathrm{P} \\
---\left(\mathrm{mg} \mathrm{dm}^{-3}\right)--- \\
\end{array}$} & \multirow{2}{*}{\multicolumn{10}{|c|}{ 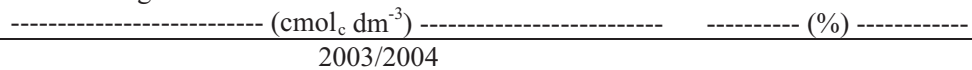 }} & \multirow[t]{2}{*}{$\begin{array}{c}\text { P-rem } \\
\left(\mathrm{mg} \mathrm{kg}^{-1}\right)\end{array}$} \\
\hline & & & & & & & & & & & & & & \\
\hline $\mathrm{PC}+\mathrm{CS}$ & 6,7 & 35,5 & 125,8 & 3,0 & 1,3 & 0,1 & 2,0 & 4,6 & 4,6 & 6,6 & 69,9 & 1,8 & 2,2 & 15,8 \\
\hline $\mathrm{TM}+\mathrm{Cr}$ & 6,7 & 39,8 & 99,5 & 3,2 & 1,3 & 0,0 & 1,8 & 4,8 & 4,8 & 6,6 & 72,4 & 0,0 & 2,2 & 15,6 \\
\hline $\mathrm{PC}$ & 7,2 & 66,1 & 160,5 & 4,2 & 1,7 & 0,0 & 1,3 & 6,3 & 6,3 & 7,6 & 82,2 & 0,0 & 2,7 & 17,2 \\
\hline $\mathrm{EB}+\mathrm{Cr}$ & 6,7 & 41,7 & 119,8 & 3,4 & 1,7 & 0,0 & 1,9 & 5,4 & 5,4 & 7,2 & 73,4 & 0,0 & 2,5 & 17,8 \\
\hline $\mathrm{PC}+\mathrm{TM}$ & 7,0 & 12,3 & 133,3 & 3,2 & 1,3 & 0,0 & 1,4 & 4,8 & 4,8 & 6,3 & 77,1 & 0,0 & 2,1 & 15,3 \\
\hline \multirow[t]{2}{*}{ Test } & 6,6 & 27,8 & 123,8 & 3,2 & 1,4 & 0,0 & 1,8 & 4,8 & 4,8 & 6,6 & 72,7 & 0,0 & 2,1 & 14,3 \\
\hline & \multicolumn{14}{|c|}{$2004 / 2005$} \\
\hline $\mathrm{PC}+\mathrm{CS}$ & 7,1 & 82,2 & 99,8 & 4,5 & 1,3 & 0,1 & 1,3 & 6,1 & 6,1 & 7,3 & 80,7 & 1,5 & 2,9 & 20,9 \\
\hline $\mathrm{TM}+\mathrm{Cr}$ & 7,0 & 62,1 & 68,3 & 4,0 & 1,1 & 0,1 & 1,6 & 5,3 & 5,4 & 7,0 & 75,5 & 1,5 & 2,8 & 21,6 \\
\hline $\mathrm{PC}$ & 7,0 & 39,8 & 204,0 & 3,6 & 1,3 & 0,1 & 1,9 & 5,5 & 5,5 & 7,3 & 75,2 & 1,8 & 2,9 & 21,6 \\
\hline $\mathrm{EB}+\mathrm{Cr}$ & 7,1 & 17,4 & 113,5 & 4,1 & 1,4 & 0,1 & 1,6 & 5,8 & 5,8 & 7,4 & 79,6 & 1,0 & 3,0 & 19,4 \\
\hline $\mathrm{PC}+\mathrm{TM}$ & 7,4 & 81,6 & 108,5 & 4,5 & 1,3 & 0,0 & 1,0 & 6,1 & 6,1 & 7,1 & 86,1 & 0,0 & 2,9 & 20,6 \\
\hline Test & 7,2 & 33,3 & 93,3 & 3,8 & 1,5 & 0,0 & 1,2 & 5,5 & 5,5 & 6,7 & 81,2 & 0,0 & 2,7 & 19,3 \\
\hline
\end{tabular}

${ }^{(1)} \mathrm{SB}$, soma de bases trocáveis; t, capacidade de troca catiônica efetiva; T, capacidade de troca catiônica pH 7; V, índice de saturação de bases; m, índice e saturação de alumínio; MO, matéria orgânica - oxidação $\mathrm{Na}_{2} \mathrm{Cr}_{2} \mathrm{O}_{7} 4 \mathrm{~N}+\mathrm{H}_{2} \mathrm{SO}_{4} 10 \mathrm{~N}$; P-rem, fósforo remanescente; PC+CS, palha de café + chorume suíno; TM+Cr, torta de mamona + crotalária; $\mathrm{PC}$, palha de café; $\mathrm{EB}+\mathrm{Cr}$, esterco bovino + crotalária; PC+TM, palha de café + torta de mamona; Test, adubação orgânica padrão. 
analisador de dossel das plantas (LAI-2000), com leituras em quatro diferentes pontos das parcelas, tendo-se obtido um valor médio de IAF de cada unidade experimental. Fez-se a colheita, em cada unidade experimental, quando os cafeeiros atingiram percentual médio de frutos verdes entre $10 \mathrm{e} 15 \%$; a produtividade (sacas ha ${ }^{-1}$ ) correspondente às safras 2003/2004 e 2004/2005 foi estimada, tendo-se considerado o rendimento médio equivalente a $20 \%$ do peso dos frutos colhidos.

Valores de incidência da cercosporiose e da ferrugem nas folhas foram transformados em área abaixo da curva de progresso da incidência (AACPI), conforme Shaner \& Finney (1977), e os índices médios da desfolha foram transformados em área abaixo da curva de progresso da desfolha (AACPDesf). Os valores de AACPI, AACPDesf, IAF e produtividade, bem como os de incidência e de severidade da cercosporiose nos frutos, foram submetidos à análise de variância pelo teste $\mathrm{F}$, cujas variáveis significativas foram submetidas ao teste de médias.

\section{Resultados e Discussão}

De acordo com as análises foliares, realizadas no estádio inicial de desenvolvimento dos frutos, denominado fase de "chumbinho", nenhum tratamento afetou significativamente os teores de $\mathrm{N}$ e $\mathrm{P}$ foliares. Todas as fontes propiciaram teores de $\mathrm{N}$ e de $\mathrm{P}$ foliares no limiar ou acima das faixas críticas de 27,8 a $31 \mathrm{~g} \mathrm{~kg}^{-1}$ e de 1,3 e $1,6 \mathrm{~g} \mathrm{~kg}^{-1}$, respectivamente, sugeridas por Martinez et al. (2003), para lavouras cafeeiras com produção acima de 30 sacas ha $^{-1}$ (produção alta), na região do Sul de Minas. O mesmo não se observou, porém, com relação ao teor de $\mathrm{K}$ foliar. Conforme se verificou no segundo ano de adubação, cafeeiros adubados unicamente com palha de café (PC) tiveram teor de K superior $(\mathrm{p} \leq 0,0197)$ aos demais tratamentos $\left(27,1 \mathrm{~g} \mathrm{~kg}^{-1}\right)$, enquanto o mais baixo teor deste nutriente $\left(24,5 \mathrm{~g} \mathrm{~kg}^{-1}\right)$ foi encontrado na torta de mamona associada à palha de café (PC+TM). Mesmo assim, em todos os tratamentos, o teor de $\mathrm{K}$ foliar na fase "chumbinho" encontrava-se dentro da faixa crítica de 20,7 a 30,5 $\mathrm{g} \mathrm{kg}^{-1}$ sugerida por Martinez et al. (2003).

O cálcio foliar analisado na fase "chumbinho", tanto em 2004 como em 2005, foi significativamente ( $\mathrm{p} \leq 0,001)$ mais baixo no tratamento PC $\left(7,4\right.$ e $9,3 \mathrm{~g} \mathrm{~kg}^{-1}$, respectivamente) comparado à testemunha $(8,6 \mathrm{e}$ $10,6 \mathrm{~g} \mathrm{~kg}^{-1}$, respectivamente) e à torta de mamona associada à crotalária $(\mathrm{TM}+\mathrm{Cr})$, cujos teores foram maiores (10,1 e 10,4 $\mathrm{g} \mathrm{kg}^{-1}$, respectivamente). Com exceção do tratamento $\mathrm{PC}$, no primeiro ano, os demais tratamentos propiciaram teor de $\mathrm{Ca}$ foliar dentro da faixa crítica $\left(8,7\right.$ a $\left.14,1 \mathrm{~g} \mathrm{~kg}^{-1}\right)$ para lavouras cafeeiras com alta produção (Martinez et al., 2003).

Quanto ao magnésio, verificaram-se valores significativamente $(\mathrm{p} \leq 0,001)$ menores no tratamento PC (2,6 $\mathrm{g} \mathrm{kg}^{-1}$ em 2004 e 2,3 $\mathrm{g} \mathrm{kg}^{-1}$ em 2005), que se diferenciou do tratamento TM+Cr $\left(3,5 \mathrm{~g} \mathrm{~kg}^{-1} \mathrm{em} 2004\right.$ e 3,2 $\mathrm{g} \mathrm{kg}^{-1}$ em 2005) com maior teor de $\mathrm{Mg}$ foliar. Nos demais tratamentos, o $\mathrm{Mg}$ encontrou-se dentro da faixa crítica (2,6 a 4,4 $\left.\mathrm{g} \mathrm{kg}^{-1}\right)$ definida para as lavouras cafeeiras, nos critérios de Martinez et al. (2003). O S não foi influenciado pelas fontes de adubação nos dois anos.

As variações nutricionais ocorridas nos cafeeiros, em conseqüência das fontes utilizadas, poderão ser melhor evidenciadas em um período mais longo de estudos, principalmente por se tratar de lavoura adubada exclusivamente com fontes orgânicas por mais de oito anos, cujas variáveis relacionadas à fertilidade de solo (Tabela 3) foram classificadas como em nível bom ou muito bom, conforme Guimarães et al. (1999). Apesar disso, os teores foliares dos nutrientes $\mathrm{K}, \mathrm{Ca}$ e $\mathrm{Mg}$ diferiram em relação às fontes de adubação, considerando-se as amostragens feitas na fase "chumbinho". Teores de nutrientes estabelecidos como faixa crítica (Martinez et al., 2003) ou faixa adequada (Malavolta et al., 1997; Guimarães et al., 1999) para a cafeicultura, normalmente, são determinados nessa fase, o que permite avaliar o estado nutricional dos cafeeiros antes do enchimento de grãos. Entretanto, no presente trabalho, observou-se redução no teor foliar dos nutrientes, da fase "chumbinho" para a fase de maturação dos frutos, conforme foi verificado em 2004 e 2005 para N (26 e 18\%), P (22 e 18\%), K (26 e 14\%), $\mathrm{Ca}(18$ e $21 \%), \mathrm{Mg}(37$ e $30 \%)$ e S (16 e $35 \%)$, considerada um dreno natural de nutrientes desviados das folhas para os frutos, durante a frutificação do cafeeiro (Matta et al., 1999; Malavolta et al., 2002; Laviola et al., 2007).

Entre os macronutrientes estudados, o Ca foliar foi o único afetado significativamente pela interação entre as fases de desenvolvimento dos frutos e as fontes de adubação ( $p \leq 0,0381)$, conforme verificado no primeiro ano de adubação. Na fase "chumbinho", o tratamento $\mathrm{PC}$ propiciou menor teor de $\mathrm{Ca}\left(7,4 \mathrm{~g} \mathrm{~kg}^{-1}\right)$ aos cafeeiros, enquanto o maior teor $\left(10,2 \mathrm{~g} \mathrm{~kg}^{-1}\right)$ foi verificado no tratamento $\mathrm{TM}+\mathrm{Cr}$. Na fase de granação, quando se registraram as maiores concentrações de $\mathrm{Ca}$ foliar nos 
tratamentos $\mathrm{TM}+\mathrm{Cr}, \mathrm{TM}+\mathrm{CC}$ e testemunha $(14,3,13,7 \mathrm{e}$ $13,7 \mathrm{~g} \mathrm{~kg}^{-1}$, respectivamente), o menor teor foi registrado no $\mathrm{EB}+\mathrm{Cr}\left(10,5 \mathrm{~g} \mathrm{~kg}^{-1}\right)$. Na fase de maturação, PC e $\mathrm{EB}+\mathrm{Cr}$ propiciaram os menores teores $\left(6,1 \mathrm{e} 5,9 \mathrm{~g} \mathrm{~kg}^{-1}\right.$, respectivamente), em contraste com $\mathrm{PC}+\mathrm{CS}$ que proporcionou o maior teor $\left(8,2 \mathrm{~g} \mathrm{~kg}^{-1}\right)$ de cálcio.

Sabe-se que o período de frutificação, durante o qual foram monitoradas as alterações nutricionais, é também aquele propício à cercosporiose e à ferrugem-docafeeiro. A análise da área abaixo da curva de progresso da incidência (AACPI) de ambas as doenças demonstrou que elas foram influenciadas pelas fontes nutricionais orgânicas testadas. O uso da palha de café, como única fonte de adubação, propiciou AACPI da cercosporiose $(\mathrm{p} \leq 0,0301)$ e da ferrugem $(\mathrm{p} \leq 0,003)$ superiores aos demais tratamentos testemunha, com exceção do $\mathrm{EB}+\mathrm{Cr}$ (Tabela 4). Comparativamente, os valores de AACPI no PC foram, respectivamente, 62 e $38 \%$ superiores à testemunha. Quando a palha de café foi combinada com torta de mamona $(\mathrm{PC}+\mathrm{TM})$ ou chorume suíno $(\mathrm{PC}+\mathrm{CS})$, houve redução da incidência da cercosporiose em $38 \%$ e da ferrugem em 31 e $21 \%$, respectivamente, com relação ao observado no tratamento PC, o que melhorou a resistência dos cafeeiros às doenças. A maior predisposição dos cafeeiros à cercosporiose e à ferrugem no PC coincidiu com os elevados teores de K e baixos teores de $\mathrm{Ca}$ e de $\mathrm{Mg}$ foliares, em comparação com as demais fontes de adubação. Em contrapartida, no $\mathrm{PC}+\mathrm{TM}$ e $\mathrm{PC}+\mathrm{CS}$, o teor de $\mathrm{K}$ foi reduzido em $27 \%$ e o teor de Ca aumentado em $15 \%$, ao final da granação, o que resultou em menor progresso das doenças, semelhante estatisticamente à testemunha, que teve menores incidências. O CS e a TM são fontes de

Tabela 4. Área abaixo da curva de progresso da incidência de cercosporiose (AACPIc) e de ferrugem (AACPIf) nas folhas e da desfolha (AACPDesf), e produtividade equivalente às safras 2004 e 2005 de cafeeiros, em diferentes fontes de adubação orgânica.

\begin{tabular}{lllllll}
\hline Tratamento $^{(2)}$ & AACPIc & AACPIf & AACPDesf & \multicolumn{3}{c}{ Produtividade $\left(\text { saca ha }^{-1}\right)^{(1)}$} \\
\cline { 5 - 7 } & & & & 2004 & 2005 & Média \\
\hline PC+CS & $5.541 \mathrm{a}$ & $8.875 \mathrm{ab}$ & $2.933 \mathrm{a}$ & $55,5 \mathrm{a}$ & $28,1 \mathrm{bc}$ & 42 \\
$\mathrm{TM}+\mathrm{Cr}$ & $6.521 \mathrm{ab}$ & $8.868 \mathrm{ab}$ & $2.844 \mathrm{a}$ & $53,0 \mathrm{a}$ & $26,6 \mathrm{bc}$ & 40 \\
$\mathrm{PC}$ & $9.046 \mathrm{~b}$ & $11.268 \mathrm{c}$ & $5.047 \mathrm{~b}$ & $43,0 \mathrm{a}$ & $9,0 \mathrm{a}$ & 26 \\
$\mathrm{~EB}+\mathrm{Cr}$ & $6.518 \mathrm{ab}$ & $10.776 \mathrm{bc}$ & $3.653 \mathrm{ab}$ & $48,0 \mathrm{a}$ & $16,6 \mathrm{ab}$ & 32 \\
PC+TM & $5.597 \mathrm{a}$ & $7.761 \mathrm{a}$ & $2.942 \mathrm{a}$ & $57,8 \mathrm{a}$ & $28,0 \mathrm{bc}$ & 43 \\
Test & $5.593 \mathrm{a}$ & $7.945 \mathrm{a}$ & $3.082 \mathrm{a}$ & $55,0 \mathrm{a}$ & $36,6 \mathrm{c}$ & 46 \\
\hline
\end{tabular}

(1)Médias seguidas por letras iguais, na coluna, não diferem entre si pelo teste de Tukey, a 5\% de probabilidade. ${ }^{(2)} \mathrm{PC}+\mathrm{CS}$, palha de café + chorume suíno; $\mathrm{TM}+\mathrm{Cr}$, torta de mamona + crotalária; PC, palha de café; $\mathrm{EB}+\mathrm{Cr}$, esterco bovino + crotalária; $\mathrm{PC}+\mathrm{TM}$, palha de café + torta de mamona; Test, adubação orgânica padrão. adubação com altos teores de $\mathrm{Ca}$, o que possibilitou a complementação do baixo teor presente na PC (Tabela 2).

Da mesma forma que nas folhas, a incidência da cercosporiose nos frutos foi influenciada pelos tratamentos nas duas safras. No primeiro ano de adubação ( $\mathrm{p} \leq 0,0163)$, o tratamento PC diferiu dos demais com a maior incidência $(25 \%)$ e a testemunha com a menor (16\%), enquanto $\mathrm{EB}+\mathrm{Cr}(21 \%), \mathrm{PC}+\mathrm{TM}(20 \%)$, $\mathrm{TM}+\mathrm{Cr}(19 \%)$ e $\mathrm{PC}+\mathrm{CS}(18 \%)$ foram estatisticamente semelhantes entre si. Resultados similares foram verificados em 2005 ( $\mathrm{p} \leq 0,0107)$. Não houve efeito significativo dos tratamentos sobre a severidade da doença nos frutos.

Os tratamentos $\mathrm{EB}+\mathrm{Cr}$ e $\mathrm{TM}+\mathrm{Cr}$ tiveram desempenho intermediário, em relação ao $\mathrm{PC}$ e à testemunha, na redução da incidência da cercosporiose e da ferrugem nas folhas (Tabela 4). Novamente, verificaram-se diferenças nos teores nutricionais foliares dos cafeeiros submetidos a esses tratamentos. A Cr associada à TM propiciou teor de Ca foliar na fase de granação do cafeeiro $35 \%$ superior ao obtido quando foi associada ao EB e estatisticamente igual ao encontrado na testemunha. O EB é considerado uma fonte de adubação pobre em $\mathrm{Ca}$ e $\mathrm{Mg}$, enquanto a TM é rica nesses nutrientes (Tabela 2), o que equilibrou a nutrição dos cafeeiros e reduziu a incidência das doenças. Embora a TM possua maior solubilidade dentre as fontes de adubação testadas, seu uso associado à crotalária deve ter reduzido eventuais perdas de nutrientes, como o $\mathrm{N} \mathrm{e} \mathrm{o} \mathrm{K}$, por volatilização ou lixiviação, responsáveis por desequilíbrios nutricionais das plantas. Adubos verdes de ciclo curto, como Crotalaria juncea, além de agirem diretamente na preservação da fertilidade do solo, produzem ácidos orgânicos que aumentam a solubilização de minerais e disponibilizam nutrientes no período mais intenso de frutificação (Ricci et al., 2005).

O desequilíbrio de nutrientes pode proporcionar maior intensidade das doenças e promover variações nos mecanismos bioquímicos e estruturais de defesa do hospedeiro (Develash \& Sugha, 1997; Rodrigues et al., 2003; Balardin et al., 2006; Basseto et al., 2007; Pratissoli et al., 2007). Doses elevadas de K, em solução nutritiva, aumentaram a incidência da cercosporiose em mudas de cafeeiros (Pozza et al., 2000). Este nutriente, de modo geral, aumenta a resistência às doenças, promove rápida recuperação dos tecidos danificados e aumento na espessura da cutícula e da parede celular, o que dificulta 
a penetração do patógeno e o progresso da infecção (Xu \& Heath, 1998). Entretanto, o excesso de K inibe a absorção de outros cátions como $\mathrm{Ca}$ e $\mathrm{Mg}$, em decorrência de competição pelos sítios de absorção ocupados pelo $\mathrm{K}$, o que favorece a cercosporiose do cafeeiro, conforme Pozza et al. (2001) e Garcia Júnior et al. (2003). O cálcio é essencial para a integridade das células, principalmente em razão da função dos poligalacturonatos de $\mathrm{Ca}$, requeridos na lamela média para a estabilidade da parede celular. Fungos produzem enzimas pectinolíticas, que destroem os poligalacturonatos durante a infecção, e colonizam os tecidos vegetais. Essas enzimas são inibidas pelo Ca (Biggs et al., 1997).

Considerando-se o dreno natural de nutrientes das folhas para o fruto, as alterações nutricionais demonstradas ao longo do período de frutificação do cafeeiro indicaram teor de Ca foliar abaixo da faixa crítica durante a fase de "chumbinho", início do progresso da cercosporiose e da ferrugem, como registrado no tratamento PC. Na fase de maturação dos frutos, quando as doenças se intensificaram, os teores eram menores ainda, sobretudo nos tratamentos $\mathrm{PC}$ e $\mathrm{EB}+\mathrm{Cr}$. Isto foi observado nos dois ciclos produtivos do cafeeiro. Em contrapartida, nos cafeeiros adubados com fontes com TM ou CS, cujo progresso da cercosporiose e da ferrugem foi menor, em comparação ao PC, verificaramse no primeiro ano, na fase de maturação, teores de $\mathrm{Ca}$ foliar de 15 e $32,7 \%$, respectivamente, superiores ao PC. No segundo ano de adubação, PC+TM e PC+CS foram 12 e $16 \%$ superiores ao $\mathrm{PC}$, respectivamente.

Com menor concentração de Ca foliar, ao final do ciclo produtivo, os cafeeiros no tratamento PC tiveram a maior incidência de ambas as doenças registradas neste estudo (Figura 1). A curva de progresso da cercosporiose, no tratamento PC, demonstrou $\mathrm{Y}_{\text {máx }}$ de 26 e $38 \%$ entre junho de 2004 e julho de 2005 , respectivamente. No tratamento testemunha, os valores foram equivalentes a 15 e $16 \%$ de incidência. O progresso da ferrugem verificado na testemunha foi maior no primeiro ano (alta carga pendente), em comparação ao segundo ano, em razão do maior dreno de carboidratos e fotoassimilados para a formação dos frutos. Mas, nos tratamentos $\mathrm{PC}$ e $\mathrm{EB}+\mathrm{Cr}$, a diferença foi mínima entre o $Y_{\text {máx }}$ da ferrugem de 2004 e 2005: 59 e $52 \%$, no PC, e de 52 e $49 \%$ no $\mathrm{EB}+\mathrm{Cr}$, em contraste com a testemunha (45 e $35 \%)$.

Com relação ao padrão vegetativo dos cafeeiros nos tratamentos, verificou-se maior percentual de desfolha no tratamento PC, cujo valor de área abaixo da curva de progresso da desfolha (AACPDesf) foi significativamente superior aos demais tratamentos $(\mathrm{p} \leq 0,025)$, exceto para $\mathrm{EB}+\mathrm{Cr}$ (Tabela 4$)$. As diferenças quanto à retenção foliar dos cafeeiros, verificadas nos tratamentos, foram confirmadas pela avaliação do índice de área foliar (IAF) no segundo ano de estudo. Entre maio e julho, ao final do período de frutificação, quando os frutos estavam na fase de maturação e ocorria a máxima intensidade da cercosporiose e da ferrugem, os valores médios de IAF variaram da seguinte forma $(\mathrm{p} \leq 0,032)$ : testemunha, 5,$6 ; \mathrm{TM}+\mathrm{Cr}, 5,6 ; \mathrm{PC}+\mathrm{CS}, 4,8$; PC+TM, 4,7; EB+Cr, 3,9; e PC, 3,9. Não foi realizada nenhuma medida de controle das doenças, por tratar-se de cafeeiros orgânicos. A pulverização com Viça-café plus, uma prática de manejo aplicada em todo o cafezal, visou principalmente o fornecimento de zinco, boro e cobre.

Além de maior desfolha, os tratamentos $\mathrm{PC}$ e $\mathrm{EB}+\mathrm{Cr}$ destacaram-se pelo efeito negativo sobre a produtividade (Tabela 4). Embora, no primeiro ano de adubação, os tratamentos tenham sido estatisticamente semelhantes, no segundo ano, esses tratamentos tiveram menor produção, tendo-se diferenciado $(\mathrm{p} \leq 0,0001)$ da testemunha, com a maior produtividade, e dos tratamentos $\mathrm{PC}+\mathrm{CS}, \mathrm{PC}+\mathrm{TM}$ e $\mathrm{TM}+\mathrm{Cr}$. É interessante analisar, também, a redução na produção dos cafeeiros de um ano para o outro, o que acentuou a bienalidade e comprometeu a safra 2005: testemunha (33,5\%), $\mathrm{PC}+\mathrm{CS}$ (49,3\%), $\mathrm{TM}+\mathrm{Cr}(49,8 \%), \mathrm{PC}+\mathrm{TM}(51,8 \%), \mathrm{EB}+\mathrm{Cr}$ $(65,3 \%)$ e PC $(78 \%)$. Esses resultados confirmam o fato de que a desfolha prematura, intensificada pela doença, além de reduzir a capacidade fotossintética dos cafeeiros, reduz o crescimento vegetativo e o tamanho dos frutos, durante o ciclo produtivo do cafeeiro. Sabe-se que em longo prazo, a intensa queda de folhas, do ciclo anterior da doença, reduz o número de nós, o que danifica a formação dos botões florais que produziriam café e reduz a capacidade produtiva do cafeeiro para a safra seguinte. A menor bienalidade, verificada na testemunha, resultou do melhor fornecimento de nutrientes, especialmente $\mathrm{K}$ e Ca foliares, por meio de suas quatro fontes nutricionais, aliado à menor intensidade da cercosporiose e da ferrugem. Deve-se considerar que a ausência de diferenças estatísticas na produtividade dos cafeeiros, entre os tratamentos no primeiro ano de adubação, está relacionada ao potencial de suprimento inicial de nutrientes do solo, rico em matéria orgânica, característico desse sistema de produção. Essas reservas podem ser liberadas e contribuir para a nutrição das plantas, o que pode ter mascarado o efeito dos tratamentos no primeiro ano de estudo. 
O presente estudo, ao analisar o progresso da cercosporiose e da ferrugem-do-cafeeiro em situação de desequilíbrio nutricional, como o proporcionado pelo tratamento PC, e em ambiente onde predomina a ausência de controle químico efetivo, demonstrou aspectos da relação entre nutrição e doenças, que normalmente é investigada sob condições controladas e em plantas infectadas. Esses aspectos ainda não tinham sido estudados em cafeeiros em produção no sistema orgânico, e os resultados corroboram a relação do potássio e do cálcio com a cercosporiose, até então relatada em mudas de café, em que os nutrientes foram fornecidos em solução nutritiva ou por meio de adubos químicos (Pozza et al., 2001; Garcia Júnior et al., 2003).

As alterações nos teores foliares dos cafeeiros adubados exclusivamente com fontes orgânicas podem ocorrer de forma diferente do observado no manejo nutricional com adubos prontamente solúveis, pois, tratase de fontes nutricionais que, ao serem aplicadas, passam por processo de mineralização antes de, lentamente, tornarem disponíveis os nutrientes às plantas. A maior ou menor eficiência na disponibilização de nutrientes para o cafeeiro está diretamente relacionada à decomposição das fontes de adubação, que é influenciada,
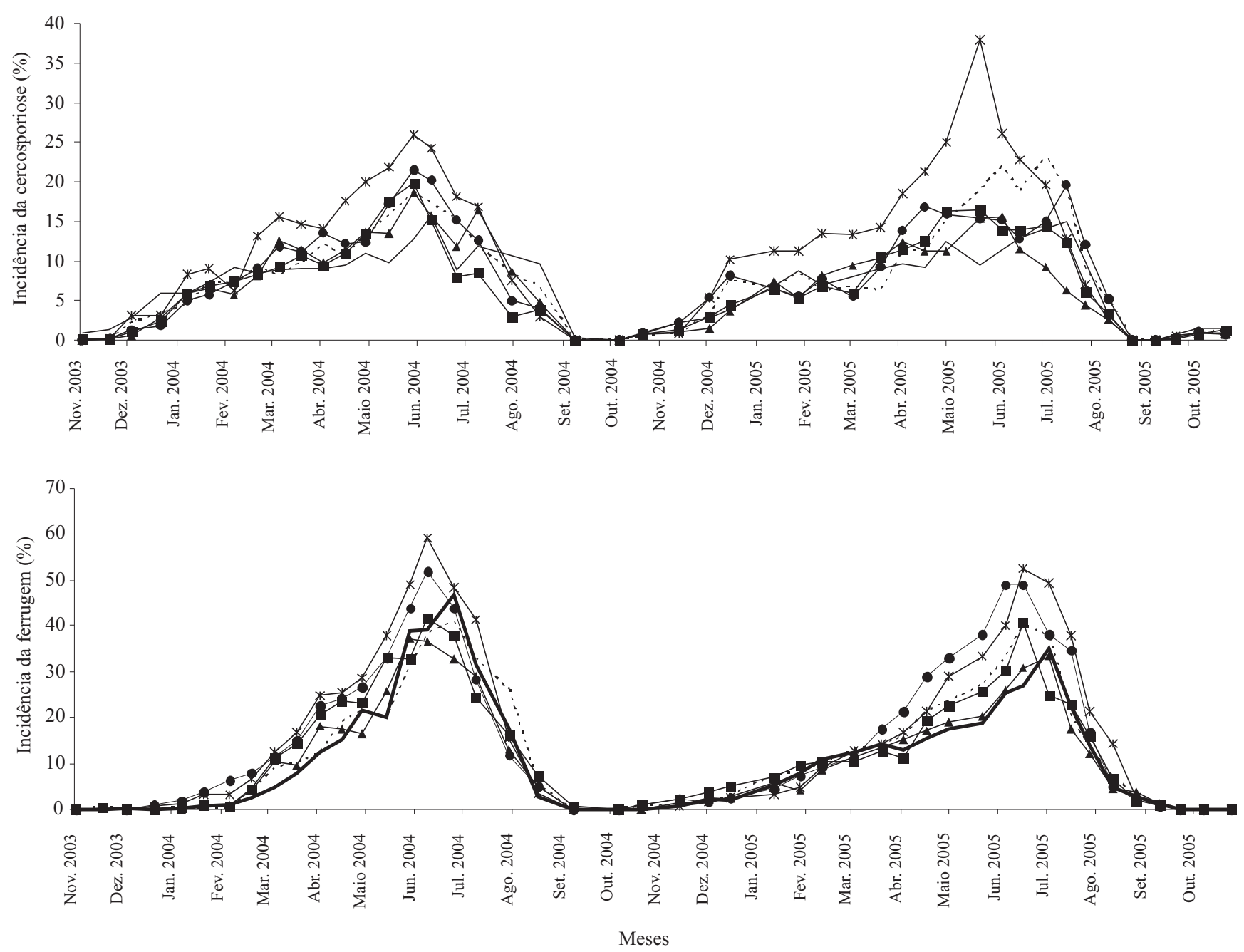

$\longrightarrow$ Test $\longrightarrow \mathrm{PC}+\mathrm{CS} \quad \cdots \cdots+\mathrm{TM}+\mathrm{Cr} \quad \longrightarrow \mathrm{EB}+\mathrm{Cr} \quad$ * $\mathrm{PC} \quad \longrightarrow \mathrm{PC}+\mathrm{TM}$

Figura 1. Progresso da incidência da cercosporiose e da ferrugem-do-cafeeiro, de 2003 a 2005, em plantas com adubação de fontes orgânicas: adubação orgânica padrão (Test); palha de café + chorume suíno (PC+CS); torta de mamona + crotalária $(\mathrm{TM}+\mathrm{Cr})$; esterco bovino + crotalária $(\mathrm{EB}+\mathrm{Cr})$; palha de café $(\mathrm{PC})$; palha de café + torta de mamona $(\mathrm{PC}+\mathrm{TM})$. 
principalmente, pela quantidade de carbono e nitrogênio (relação C:N) do material, além de outros fatores como o manejo, a temperatura e a umidade do solo. A maior ou menor relação $\mathrm{C}: \mathrm{N}$ pode causar imobilização ou lixiviação dos nutrientes, portanto, deve-se procurar associar materiais que propiciem uma sincronização entre a liberação de nutrientes e a demanda do cafeeiro (Ricci et al., 2005).

A definição de um manejo adequado, para a adubação dos cafeeiros orgânicos, reduz o impacto causado pela cercosporiose e pela ferrugem, o que garante redução na perda de folhas e ramos e melhor produtividade, possivelmente, pela diluição do efeito da bienalidade nas safras. $\mathrm{O}$ uso de duas fontes de adubação, muitas vezes, teve efeito semelhante ao tratamento testemunha com cinco fontes nutricionais. Isto pode diminuir custos, a depender da disposição do material na lavoura. A palha de café, resíduo presente na lavoura cafeeira, combinada com a torta de mamona ou com chorume suíno consistiu tratamentos que, em todas as variáveis analisadas, tiveram desempenho estatisticamente semelhante à testemunha, considerada adubação padrão da fazenda. Os efeitos dos tratamentos, no primeiro ano de adubação, podem ter sido reduzidos pelo fato de os cafeeiros estarem ainda sob influência da adubação-padrão do ano agrícola anterior. Assim, evidencia-se a necessidade de se manterem ensaios no campo, por tempo mais prolongado do que os dois anos durante os quais se procedeu à esta investigação, para averiguar se as tendências observadas no presente estudo se confirmam.

\section{Conclusões}

1. O progresso da cercosporiose e da ferrugem é maior com a fonte palha de café, que eleva os teores de $\mathrm{K}$ e reduz os teores de $\mathrm{Ca}$ e $\mathrm{Mg}$ nas folhas dos cafeeiros.

2. Menores concentrações de $\mathrm{K}$ e maiores de $\mathrm{Ca}$ nas folhas, durante a fase de granação, conferem maior equilíbrio nutricional aos cafeeiros, aumentam a resistência à cercosporiose e à ferrugem e reduzem a desfolha e o efeito de bienalidade na produção.

3. Palha de café, combinada com torta de mamona ou com chorume suíno, propiciam incidência, enfolhamento e produtividade do cafeeiro semelhantes à testemunha com quatro fontes nutricionais (composto orgânico + torta de mamona + chorume suíno ou farinha de ossos e carne + crotalária).

\section{Agradecimentos}

À Fundação de Amparo à Pesquisa de Minas Gerais, pelo financiamento da pesquisa; aos proprietários da Fazenda Cachoeira, pelo apoio na instalação e condução dos experimentos; ao Sr. Ivan Franco Caixeta, representante da Associação de Cafeicultura Orgânica do Brasil, pela parceria e pelo apoio logístico nas normas de produção de café orgânico; à Coordenação de Aperfeiçoamento de Pessoal de Nível Superior, por concessão da bolsa.

\section{Referências}

ACUÑA, R.S.; ZAMBOLIM, L.; CRUZ, C.D.; VALE, F.X.R. Estudo epidemiológico da ferrugem-do-cafeeiro (Hemileia vastatrix) utilizando a análise de trilha. Fitopatologia Brasileira, v.23, p.425-430, 1998.

BALARDIN, R.S.; DALLAGNOL, L.J.; DIDONÉ, H.T.; NAVARINI, L. Influência do fósforo e do potássio na severidade da ferrugem-da-soja Phakopsora pachyrhizi. Fitopatologia Brasileira, v.31, p.462-467, 2006.

BASSETO, M.A.; CERESINI, P.C.; VALÉRIO FILHO, W.V. Severidade da mela-da-soja causada por Rhizoctonia solani AG-1 IA em função de doses de potássio. Summa Phytopathologica, v.33, p.56-62, 2007.

BIGGS, A.R.; EL KHOLI, M.M.; EL NESHAWY, S.; NICKERSON, R. Effects of calcium salts on growth, polygalacturonase activity, and infection of peach fruit by Monilinia fructicola. Plant Disease, v.81, p.399-403, 1997.

BOLDINI, J.M. Epidemiologia da ferrugem e da cercosporiose do cafeeiro (Coffea arabica L.) irrigado e fertirrigado por gotejamento. 2001. 68p. Tese (Dissertação) - Universidade Federal de Lavras, Lavras.

BRUGGEN, A.H.C. Plant disease severity in high-input compared to reduced-input and organic farming systems. Plant Disease, v.79, p.976-984, 1995.

CARVALHO, V.L.; CHALFOUN, S.M. Manejo integrado das principais doenças do cafeeiro. Informe Agropecuário, v.19, p.27-35, 1998.

CARVAlHO, V.L.; CHALFOUN, S.M.; CASTRO, H.A.; CARVALHO, V.D. Influência de diferentes níveis de produção sobre a evolução da ferrugem-do-cafeeiro e sobre teores foliares de compostos fenólicos. Ciência e Agrotecnologia, v.25, p.49-54, 2001.

EMBRAPA. Relatório do Consórcio Brasileiro de Pesquisa e Desenvolvimento do Café. 2004. Brasília: Embrapa Café, 2004. $36 \mathrm{p}$.

EMBRAPA. Sistema brasileiro de classificação de solos. 2.ed. Rio de Janeiro: Embrapa Solos, 2006. 306p.

DEVELASH, R.K.; SUGHA, S.K. Factors affecting development of downy mildew (Peronospora destructor) of onion (Allium cepa). Indian Journal of Agricultural Sciences, v.67, p.71-74, 1997. 
GARCIA JÚNIOR, D.; POZZA, E.A.; POZZA, A.A.A.; SOUZA, P.E.; CARVALHO, J.G.; BALIEIRO, A.C. Incidência e severidade da cercosporiose-do-cafeeiro em função do suprimento de potássio e cálcio em solução nutritiva. Fitopatologia Brasileira, v.28, p.286291, 2003.

GUIMARÃES, P.T.G.; GARCIA, A.W.R.; ALVAREZ VENEGAS, V.H.; PREZOTTI, L.C.; VIANA, A.S.; MIGUEL, A.S.; MALAVOLTA, E.; CORRÊA, J.B.; LOPES, A.S.; NOGUEIRA, F.D.; MONTEIRO, A.V.C.; OLIVEIRA, J.A. Cafeeiro. In: RIBEIRO, A.C.; GUIMARÃES, P.T.G.; ALVAREZ VENEGAS, V.H. (Ed.). Recomendações para uso de corretivos e fertilizantes em Minas Gerais: 5a aproximação. Viçosa: UFV, 1999. p.289-302.

LAVIOLA, B.G.; MARTINEZ, H.E.P.; SOUZA, R.B.; ALVAREZ VENEGAS, V.H. Dinâmica de cálcio e magnésio em folhas e frutos de Coffea arabica. Revista Brasileira de Ciência do Solo, v.31, p.319-329, 2007.

MALAVOLTA, E.; FAVARIN, J.L.; MALAVOLTA, M.; CABRAL, C.P.; HEINRICHS, R.; SILVEIRA, J.S.M. Nutrients repartition in the coffee branches, leaves and flowers. Pesquisa Agropecuária Brasileira, v.37, p.1017-1022, 2002.

MALAVOLTA, E.; VITTI, G.C.; OLIVEIRA, S.A. Avaliação do estado nutricional das plantas: princípios e aplicações. Piracicaba: Potafos, 1997. 238p.

MARTINEZ, H.E.P.; MENEZES, J.F.S.; SOUZA, R.B.; ALVAREZ VENEGAS, V.H.; GUIMARÃES, P.T.G. Faixas críticas de concentrações de nutrientes e avaliação do estado nutricional de cafeeiros em quatro regiões de Minas Gerais. Pesquisa Agropecuária Brasileira, v.38, p.703-713, 2003.

MARTINS, M.; MENDES, A.N.G.; ALVARENGA, M.I.N. Incidência de pragas e doenças em agroecossistemas de café orgânico de agricultores familiares em Poço Fundo, MG. Ciência e Agrotecnologia, v.28, p.1306-1313, 2004.

MATTA, F.M.; AMARAL, J.A.T.; RENA, A.B. Growth periodicity in trees of Coffea arabica L. in relation to nitrogen supply and nitrate reductase activity. Field Crops Research, v.60, p.223-229, 1999.
PHIRI, N.A.; HILLOCKS, R.J.; JEFFRIES, P. Incidence and severity of coffee diseases in smallholder plantations in Northern Malawi. Crop Protection, v.20, p.325-332, 2001.

POZZA, A.A.A.; MARTINEZ, H.E.P.; CAIXETA, S.L.; CARDOSO, A.A.; ZAMBOLIM, L.; POZZA, E.A. Influência da nutrição mineral na intensidade da mancha-de-olho-pardo em mudas de cafeeiro. Pesquisa Agropecuária Brasileira, v.36, p.53-60, 2001.

POZZA, A.A.A.; MARTINEZ, H.E.P.; POZZA, E.A.; CAIXETA, S.L.; ZAMBOLIM, L. Intensidade da mancha-de-olho-pardo em mudas de cafeeiro em função de doses de $\mathrm{N}$ e de $\mathrm{K}$ em solução nutritiva. Summa Phytopathologica, v.26, p.29-33, 2000.

PRATISSOLI, D.; ALMEIDA, G.D.; JESUS JÚNIOR, W.C.; VICENTINI, V.B.; HOLTZ, A.M.; COCHETO, J.G. Fertilizante organomineral e argila silicatada como indutores de resistência à varíola do mamoeiro. Idesia, v.25, p.63-67, 2007.

RICCI, M.S.F.; ALVES, B.J.R.; MIRANDA, S.C.M.; OLIVEIRA, F.F. Growth rate and nutritional status of an organic coffee cropping system. Scientia Agricola, v.62, p.138-144, 2005.

RODRIGUES, F.A.; BENHAMOU, N.; DATNOFF, L.E.; JONES, J.B.; BÉLANGER, R.R. Ultrastuctural and cytochemical aspects of silicon-mediated rice blast resistance. Phytopathology, v.93, p.535-546, 2003.

SAMAYOA JUÁREZ, J.O.; SÁNCHEZ-GARCIA, V.G. Enfermedades foliares en café orgânico y convencional. Manejo Integrado de Plagas, v.58, p.9-19, 2000.

SHANER, G.; FINNEY, R.E. The effect of nitrogen fertilization on the expression of slow mildewing resistance in Knox wheat. Phytopathology, v.67, p.1051-1056, 1977.

TALAMINI, V.; SOUZA, P.E.; POZZA, E.A.; SILVA, A.M.; BUENO FILHO, J.S.S. Progresso da ferrugem e da cercosporiosedo-cafeeiro (Coffea arabica L.) em diferentes lâminas de irrigação e diferentes parcelamentos de adubação. Ciência e Agrotecnologia, v.25, p.55-62, 2001.

XU, H.; HEATH, M.C. Role of calcium in signal transduction during the hypersensitive response caused by basidiospore-derived infection of the cowpea rust fungus. Plant Cell, v.10, p.585-598, 1998.

Recebido em 4 de dezembro de 2007 e aprovado em 17 de junho de 2008 